\title{
Potentiation of androgen receptor transcriptional activity by inhibition of histone deacetylation - rescue of transcriptionally compromised mutants
}

\author{
C G Korkmaz ${ }^{* 1,2}$, K Frønsdal ${ }^{* 1,2}$, Y Zhang ${ }^{1}$, P I Lorenzo ${ }^{1}$ and \\ F Saatcioglu ${ }^{1,2,3}$ \\ ${ }^{1}$ Department of Molecular Biosciences, University of Oslo, Postboks 1041 Blindern, 0316 Oslo, Norway \\ ${ }^{2}$ Biotechnology Centre of Oslo, University of Oslo, Postboks 1041 Blindern, 0316 Oslo, Norway \\ ${ }^{3}$ Institute for Clinical Medicine, University of Oslo, Postboks 1041 Blindern, 0316 Oslo, Norway \\ (Requests for offprints should be addressed to F Saatcioglu; Email: fahris@bio.uio.no) \\ ${ }^{*}$ (C G Korkmaz and K Frønsdal contributed equally to this work)
}

\begin{abstract}
Androgens are critical in the development and maintenance of the male reproductive system and important in the progression of prostate cancer. The effects of androgens are mediated by the androgen receptor (AR), which is a ligand-modulated transcription factor that belongs to the nuclear receptor superfamily. We and others have previously shown that CREB-binding protein (CBP) can function as a coactivator for AR. Similar to some other nuclear receptor coactivators and/or the proteins that they interact with, CBP has histone acetyl transferase (HAT) activity that is thought to contribute to transcriptional activation by nuclear receptors. We have therefore assessed whether an increase in the histone acetylation status in the cell can influence AR transcriptional activity, by using the histone deacetylase (HDAC) inhibitors (HDACIs) trichostatin A (TSA), sodium butyrate (Na-But) and depsipeptide (FR901228). We found that inhibition of HDAC activity significantly increased the ability of endogenous AR in LNCaP cells, or ectopically expressed AR in HeLa cells, to activate transcription from AR-
\end{abstract}

dependent reporter constructs. In addition, HDACIs increased the androgen-dependent activation of the prostate-specific antigen (PSA) gene in LNCaP cells, an increase that was not due to an increase in nuclear AR protein levels. Moreover, the viral oncoprotein E1A that inhibits CBP HAT activity fully repressed the ability of HDACIs to stimulate AR-mediated transcription, indicating that $\mathrm{CBP}$ is involved in this process. Deletional mutagenesis of $\mathrm{AR}$ indicated that whereas the $\mathrm{AF}-2$ domain in the $\mathrm{C}$-terminus is dispensable, the $\mathrm{AF}-1$ domain in the $\mathrm{N}$-terminus is required for augmentation of $\mathrm{AR}$ action by HDACIs, an observation which is in concordance with the reduced ability of CBP to activate AR $\mathrm{N}$-terminal deletion mutants. Furthermore, HDACI treatment rescued the deficiency in the transactivation potential of AF-2 mutants. Taken together, our findings suggest that a change in the level of histone acetylation of target genes is an important determinant of AR action, possibly mediated by CBP.

Journal of Endocrinology (2004) 182, 377-389

\section{Introduction}

The androgen receptor (AR) is a member of the nuclear receptor family and mediates the effects of androgens, which are important in the development and maintenance of male sexual characteristics and prostate cancer progression (Lindzey et al. 1994, Quigley et al. 1995). Upon ligand binding, nuclear receptors bind specific DNA response elements through a highly conserved DNAbinding domain and mediate transcriptional activation through transactivating domains, referred to as activation function-1 (AF-1) and activation function-2 (AF-2), localized in the N-terminus (NTD) and the ligand-binding domain (LBD) within the C-terminus respectively (Tsai \&
O’Malley 1994, Mangelsdorf et al. 1995). Similar to some other nuclear receptors, interactions between the NTD and the LBD, mediated by coactivators, are required for AR transcriptional activity (McInerney et al. 1996, Doesburg et al. 1997, Ikonen et al. 1997). It was previously shown that CREB-binding protein (CBP) acts as a coactivator for AR through interactions with both the NTD and the LBD (Aarnisalo et al. 1998, Frønsdal et al. 1998). In addition, CBP exhibits histone acetyl transferase (HAT) activity (Bannister \& Kouzarides 1996, Ogryzko et al. 1996) and is an essential coactivator for many different classes of transcription factors in addition to nuclear receptors (Goodman \& Smolik 2000, Chan \& La Thangue 2001). 
Nuclear receptors have been proposed to regulate gene expression through association with HATs for activation and histone deacetylases (HDACs) for repression (for reviews, see Glass \& Rosenfeld 2000, McKenna \& O'Malley 2002). It is thought that ligand binding, through the recruitment of HAT-containing coactivators, such as $\mathrm{CBP}$, induces hyperacetylation on the promoter of target genes (Jenster et al. 1997). In addition, recent studies indicate that CBP and its close relative p300 can directly acetylate transcriptional regulatory proteins, such as GATA-1 (Hung et al. 1999) and P53 (Gu \& Roeder 1997, Sakaguchi et al. 1998), and modulate their activities. Recent studies suggest that nuclear receptors are directly acetylated themselves by the HAT-containing proteins at critical sites governing hormone-dependent transactivation, thereby modulating their transcriptional activity, including the AR (e.g. Cress \& Seto 2000, Gaughan et al. 2002, Fu et al. 2003).

In order to study the possible role of HAT activity in nuclear receptor function, HDAC inhibitors (HDACIs) have been used (Marks et al. 2000), since no HAT inhibitor is yet available. In this study, we utilized three different HDACIs: trichostatin A (TSA) (Tsuji et al. 1976, Yoshida et al. 1990), sodium butyrate (Na-But) (Newmark et al. 1994) and FR901228 (or depsipeptide) (Kramer et al. 2001) in order to assess the role of acetylation in AR-mediated transcription. All three HDACIs augmented androgen-mediated transcription and the AR target gene prostate-specific antigen (PSA) mRNA levels in androgen-treated $\mathrm{LNCaP}$ cells. We found that the ability of CBP to act as a coactivator for different AR mutants correlated with the response of the mutants to different HDACIs. In addition, E1A, a specific inhibitor of $\mathrm{CBP}$, abolished AR activity in the presence or absence of HDACIs. These results provide evidence that an increase in histone acetylation of target genes is essential for mediating AR transcriptional activity.

\section{Materials and Methods}

\section{Cell culture, transient transfection and luciferase assays}

HeLa cells were maintained in Dulbecco's modified Eagle's medium (DMEM) supplemented with 5\% fetal bovine serum (FBS) (Gibco-BRL), glutamine, penicillin and streptomycin. LNCaP cells were obtained from the American Type Culture Collection (ATCC) (Manassas, VA, USA) and cultured in RPMI 1640 medium (Gibco-BRL) which was supplemented with $10 \%$ FBS, glutamine, penicillin and streptomycin.

The calcium phosphate coprecipitation method (Frønsdal et al. 2000) was used to transfect both the HeLa and LNCaP cells. Transfection of HeLa cells was carried out in $12-$ well plates $\left(6 \cdot 0 \times 10^{4}\right.$ cells/well), with $0 \cdot 25 \mu \mathrm{g}$ reporter plasmid, indicated amounts of AR expression vector or empty expression vector and pUC18 to a total of
$1 \mu \mathrm{g}$ DNA per well. Transfection of LNCaP cells was carried out in six-well plates in $3 \cdot 0 \times 10^{5}$ cells/well, with $0.67 \mu \mathrm{g}$ reporter plasmid and pUC18 to a total of $2 \mu \mathrm{g}$ DNA. At $1 \mathrm{~h}$ before transfection, the medium was changed with DMEM supplemented with 5\% FBS. At $4.5 \mathrm{~h}$ after transfection, the medium was removed, and cells were permeabilized in 15\% glycerol in PBS for $2 \mathrm{~min}$, and then washed with PBS. Cells were then fed with DMEM or RPMI, for HeLa and LNCaP cells respectively, containing $0.5 \%$ charcoal-treated FBS. R1881 (NEN), TSA (Sigma), Na-But (Sigma) and/or FR901228 (kindly provided by Dr Robert Schultz, and the National Institutes of Health, Bethesda, MD, USA) were added, as indicated, and after $18 \mathrm{~h}$, cells were washed once with cold PBS and harvested in Tris-MES solution (1 mM DTT, $0 \cdot 5 \%$ TritonX-100, $50 \mathrm{mM}$ Tris-MES $\mathrm{pH} 7 \cdot 8$ ), and luciferase activities were determined.

\section{Plasmids}

Reporter plasmids -285-PB-LUC and 2XARE-LUC (Ikonen et al. 1997) and expression vectors CMV-CBP (Kwok et al. 1994) and pBL9-E1A (Deleu et al. 2001) have been described. The expression vectors pSG5-AR (Frønsdal et al. 1998), the C-terminal deletion mutants (Frønsdal et al. 1998), C-terminal point mutations (Slagsvold et al. 2000) and N-terminal deletion mutants of AR (Slagsvold et al. 2000) have previously been described.

\section{Northern blot analysis}

Cells were maintained as above and treated with R1881 $\left(10^{-7} \mathrm{M}\right)$ together with increasing amounts of TSA, Na-But and FR901228, as indicated, for 18-20 h. Total RNA was extracted and fractionated by standard procedures. An amount of $15 \mu \mathrm{g}$ total RNA was run per lane. The probes were generated by random priming and had a specific activity of $>3 \times 10^{8}$ d.p.m. $/ \mu \mathrm{g}$. The following probes were used. For PSA, an EcoRI fragment spanning the full-length cDNA (Lundwall \& Lilja 1987) was used; for beta-actin cDNA (Clontech, Palo Alto, CA, USA), and for human glyceraldehyde-3-phosphate dehydrogenase (GAPDH), a BamHI-Xhol fragment of the cDNA was used. The bands were visualized by phosphoimager analysis (Amersham Biosciences).

\section{GST pull-down assay-in vitro}

Interactions between $\mathrm{AR}$ or its mutants and $\mathrm{CBP}$ were examined by the GST pull-down assay, as described previously (Slagsvold et al. 2000). Briefly, GST and GST-CBP (amino acids 1-452) fusion proteins were expressed in Escherichia coli and purified on glutathioneSepharose beads (Amersham Pharmacia Biotech). AR and its mutants were translated in vitro by the TNT-coupled transcription/translation system (Promega) in the presence 
of $\left[{ }^{35} \mathrm{~S}\right]$-methionine. The translated proteins were incubated on ice for $30 \mathrm{~min}$ in the presence or absence of R1881 $\left(10^{-6} \mathrm{M}\right)$ before the addition of GST or GSTCBP. The reactions were incubated for $1-2 \mathrm{~h}$ on ice in NETN buffer (40 mM NaCl, $20 \mathrm{mM}$ Tris-HCl, pH 8.0, 0.01\% IGEPAL and $1 \mathrm{mM}$ EDTA) containing protease inhibitors and R1881 $\left(10^{-6} \mathrm{M}\right)$ with occasional mixing. The beads were then washed three times with NETN buffer, resuspended in SDS-PAGE sample buffer, and size fractionated on an 8\% SDS-PAGE. After autoradiography, relative intensity of the bands was determined by densitometry.

\section{Western analysis}

Preparation of the whole cell extracts and Western analysis were done as previously described (Xi et al. 2004). AR-specific antiserum (used 1:1000 dilution) was raised against the N-terminus of human AR (aa 1-504) fused to GST. After autoradiography, relative intensity of the bands was determined by densitometry. For checking the levels of AR in response to different HDACIs, HeLa cells were cultured and $5 \mu \mathrm{g}$ of pSG5-AR was transfected in $10 \mathrm{~cm}$ dishes using Fugene (Roche). Cells were then treated with the HDACIs and R1881 as described above for $24 \mathrm{~h}$. Preparation of whole cell extracts and Western analysis were as above.

\section{Results}

Histone deacetylase inhibitors potentiate transcription by the $A R$

To determine whether the histone acetylation status of the cell affects AR function, we first performed a series of transient transfection experiments in the androgenresponsive prostate cancer cell line $\mathrm{LNCaP}$ (Horoszewicz et al. 1983). An androgen-dependent reporter construct in which a deletion derivative of the rat probasin gene promoter is fused to the luciferase (LUC) gene (-285-PBLUC) (Ikonen et al. 1997) was transfected into LNCaP cells. After transfection, the cells were either left untreated or treated with the synthetic androgen R1881 in the presence or absence of increasing concentrations of TSA, the most commonly used HDACI (Yoshida et al. 1990). The cells were harvested $18 \mathrm{~h}$ later and LUC activities were determined. As shown in Fig. 1A, R1881 treatment increased reporter expression 70-fold, and this was further potentiated in the presence of TSA, reaching approximately fourfold higher levels than with R1881 alone.

To examine further the effect of acetylation on ARmediated transcription, we tested another HDACI, sodium butyrate (Na-But), under the same conditions as for TSA. As shown in Fig. 1B, R1881 treatment alone increased -285-PB-LUC activity by 50 -fold compared with the basal level. In the presence of increasing amounts of $\mathrm{Na}-\mathrm{But}$, however, the activation by R1881 was further increased in a dose-dependent manner, reaching threefold higher levels than those obtained in the absence of Na-But.

To confirm the validity of the findings above, we tested a third HDACI, a more recently discovered compound, FR901228 (FR) (Kramer et al. 2001), that is structurally unrelated to TSA or Na-But. Transient transfection experiments were carried out in LNCaP cells, as described for TSA and Na-But. When $\mathrm{LNCaP}$ cells were transfected with -285-PB-LUC (Fig. 1C), similar to the findings above, -285-PB-LUC expression was increased approximately 10-fold in the presence of R1881 alone. In the presence of increasing amounts of FR 901228, the R1881induced levels of -285-PB-LUC expression increased by approximately $2 \cdot 5$-fold, whereas the basal level of reporter expression was not affected.

We next assessed whether the effect of HDACIs on AR function in LNCaP cells could be due to changes in AR expression. LNCaP cells were grown in the presence or absence of the HDACIs and/or R1881, and Western analysis was performed on whole-cell extracts with an AR-specific antiserum. As shown in Fig. 1D, in untreated cells R1881 treatment slightly increased the steady-state levels of AR. However, HDACI treatment did not significantly affect AR levels, suggesting instead that HDACIs influence AR function.

Similar experiments were performed in HeLa cells in the presence of ectopically expressed AR. -285-PB-LUC was cotransfected into HeLa cells either with an empty expression vector (pSG5) or an expression vector encoding AR (pSG5-AR). After transfection, cells were either left untreated or treated with the synthetic androgen R1881 in the presence or absence of increasing concentrations of TSA or Na-But. As shown in Fig. 2A, whereas there was modest activation of the reporter in the presence of AR and R1881, this was dramatically increased in the presence of increasing amounts of TSA, reaching approximately 200-fold activation. In contrast, reporter activity remained low in the absence of AR in response to TSA treatment. When Na-But was used in a similar experiment, -285PB-LUC was activated by twofold by R1881 treatment, reaching approximately 35 -fold higher levels in response to Na-But (Fig. 2B). At the highest level of $\mathrm{Na}$-But used, however, the basal level of -285-PB-LUC expression also significantly increased, although still retaining approximately sevenfold the R1881 response. These data suggest that the effects of HDAC inhibitors are not cell type specific and are likely to be directly mediated through effects on AR function. Similar results were obtained using another AR dependent reporter, 2 XARE-LUC (data not shown).

We next assessed whether the effect of HDACIs on AR function in HeLa cells at the -285-PB-LUC reporter could be due to changes in AR expression levels. HeLa cells were transfected as above and grown in the presence or 
A

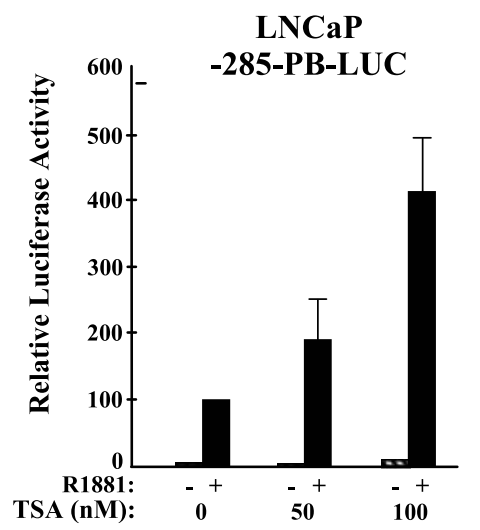

C

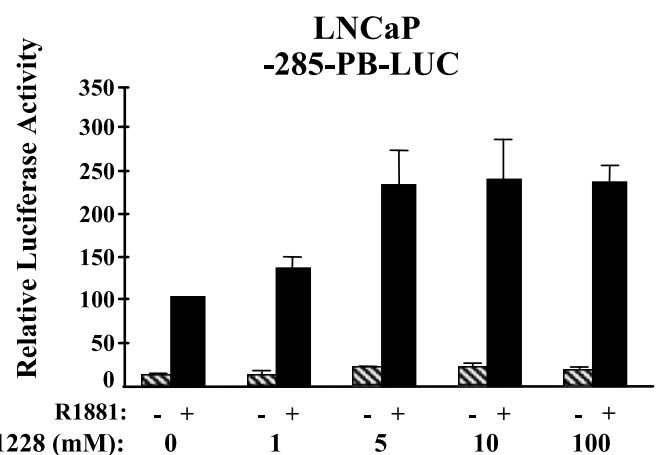

FR901228 (mM):
B
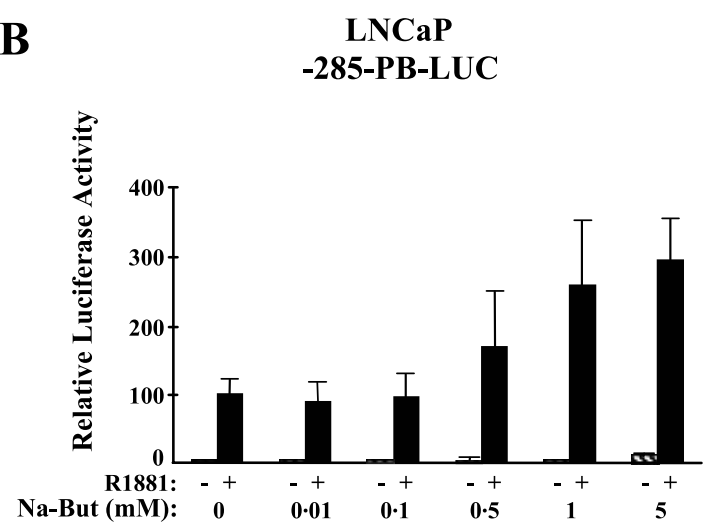

D

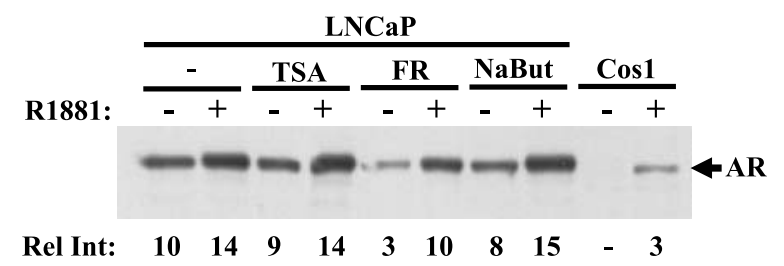

Figure 1 Effect of HDACls on AR-dependent transcriptional activation in LNCaP cells. (A) LNCaP cells were transfected with the -285-PB-LUC reporter. Cells were either left untreated or treated with R1881 $\left(10^{-9} \mathrm{M}\right)$ and/or with the indicated concentrations of TSA and harvested after $18 \mathrm{~h}$, and LUC activities were determined. Activation of reporter gene expression in the presence of R1881, but in the absence of TSA, was arbitrarily set at 100 . The differences observed were statistically significant (Mann-Whitney test, $P<0 \cdot 004)$.

(B) LNCaP cells were transfected with the -285-PB-LUC reporter. Cells were either left untreated or treated with R1881 (10-7 M) and/or with the indicated concentrations of Na-But and harvested after $18 \mathrm{~h}$, and LUC activities were determined. Activation of reporter gene expression in the presence of R1881, but in the absence of Na-But, was arbitrarily set at 100 . The differences observed were statistically significant (Mann-Whitney test, $P<0 \cdot 05)$. (C) LNCaP cells were transfected with the -285-PB-LUC reporter. Cells were either left untreated or treated with R1881 (10-7 M) and/or with the indicated concentrations of FR901228 and harvested after $18 \mathrm{~h}$, and LUC activities were determined. Activation of reporter gene expression in the presence of R1881, but in the absence of FR901228, was arbitrarily set at 100 . The differences observed were statistically significant (Mann-Whitney test, $P<0 \cdot 05$ ). (D) LNCaP cells were cultured in the presence or absence of R1881 for $24 \mathrm{~h}$, with or without the HDACls (TSA, $100 \mathrm{nM}$; Na-But, $1 \mathrm{mM}$; FR901228, $10 \mathrm{ng} / \mathrm{ml}$ ) as indicated. Whole-cell extracts were then prepared and used in Western analysis with an AR-specific antibody. Extracts made from Cos 1 cells that were either transfected with the empty expression vector pSG5 (-) or transfected with an AR-expression plasmid pSG5-AR (+) were used as controls. The data shown are representative of two independent experiments.

absence of the HDACIs and/or R1881. Whole-cell extracts were prepared and Western analysis was performed with an AR-specific antiserum. As shown in Fig. 2C, whereas there was no significant change in AR levels in the presence of TSA compared with nontreated cells, surprisingly, both Na-But and FR901228 significantly increased AR expression. This increase was reduced by approximately $10-30 \%$ in the presence of R1881. These data suggest that the effects of $\mathrm{Na}-\mathrm{But}$ and FR901228 on AR activity may in part be due to effects on AR expression.

\section{Histone deacetylase inhibitors stimulate PSA expression}

To examine more directly the possible role of HDAC inhibition on AR activity, we examined the expression of an AR target gene, PSA (Young et al. 1995) in the presence or absence of HDACIs. LNCaP cells were either left untreated or treated with R1881 and/or increasing concentrations of TSA, Na-But or FR901228 for $24 \mathrm{~h}$. Total RNA was then extracted and used in Northern blot analysis with PSA as probe. As shown in Fig. 3A, whereas the basal level of PSA mRNA accumulation was negligi- 
A

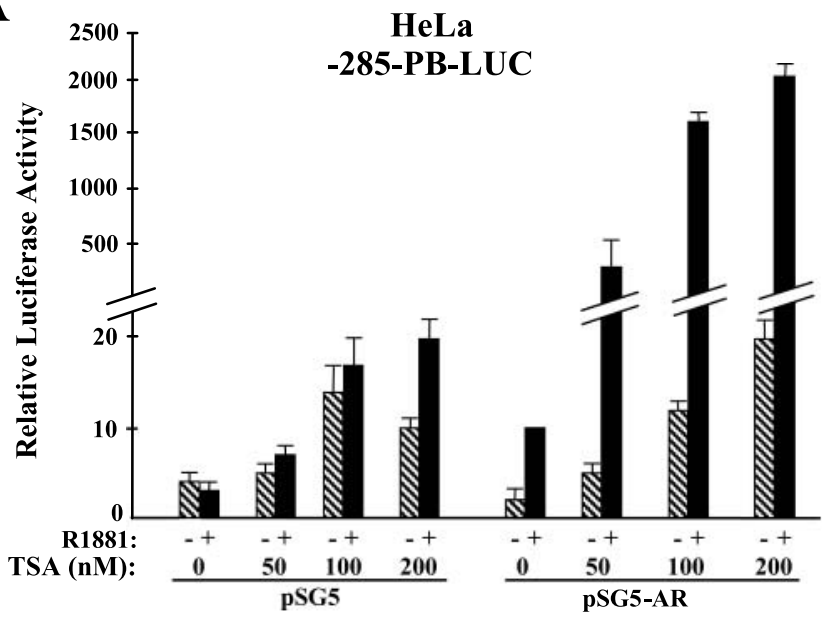

B

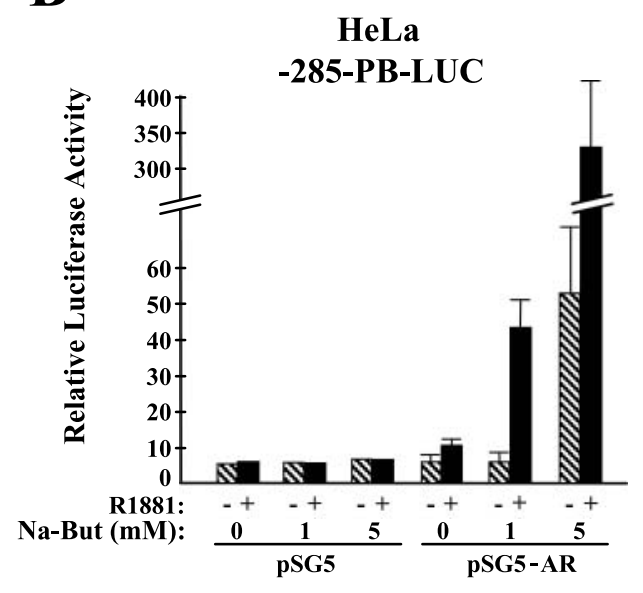

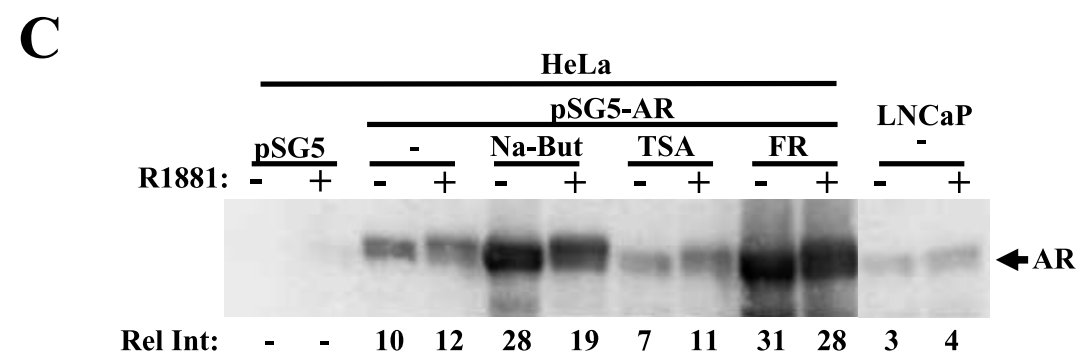

Figure 2 Effect of HDACls on AR-dependent transcriptional activation in HeLa cells. (A) HeLa cells were transfected with the -285-PB-LUC reporter in the presence of the empty expression vector pSG5 (2.9 ng) or pSG5-AR, which specifies AR production (5 ng). The different amounts for pSG5 and pSG5-AR reflect equalization of molar amounts. Cells were either left untreated or treated with R1881 $\left(10^{-9} \mathrm{M}\right)$ and/or with the indicated concentrations of TSA and harvested after $18 \mathrm{~h}$, and LUC activities were determined. Activation of reporter gene expression in the presence of pSG5-AR and R1881, but in the absence of TSA, was arbitrarily set at 10. Note that a suboptimal level of AR was used to clarify the TSA effect. The differences observed were statistically significant (Mann-Whitney test, $P<0 \cdot 05)$. (B) HeLa cells were transfected with the -285-PB-LUC reporter in the presence of cotransfected pSG5 (5 ng) or pSG5-AR (10 ng). Activation of reporter gene expression in the presence of pSG5-AR and R1881, but in the absence of Na-But, was arbitrarily set at 10. The differences observed were statistically significant (Mann-Whitney test, $P<0 \cdot 05)$. (C) HeLa cells were transfected with pSG5 or pSG5-AR (5 $\mu$ g) and were either left untreated or were treated with R1881 $\left(10^{-8} \mathrm{M}\right)$ for $24 \mathrm{~h}$ in the presence or absence of the HDACls (TSA, $100 \mathrm{nM}$; Na-But, $1 \mathrm{mM}$; FR901228, $10 \mathrm{ng} / \mathrm{ml}$ ) as indicated. Whole-cell extracts were then prepared and used in Western analysis with an AR-specific antibody. In parallel, LNCaP cells were cultured in the presence or absence of R1881 for $24 \mathrm{~h}$, and whole-cell extracts were prepared and used in Western analysis as above. The experiment was repeated three times with consistent results. The results of densitometric analysis are shown below the Figure as relative intensity (Rel. Int.).

ble, it dramatically increased (approximately by 10 -fold) in response to hormone. Upon treatment with increasing concentrations of TSA, hormone-induced levels of PSA mRNA accumulation were increased in a dose-dependent manner, reaching 3·2-fold higher levels than with R1881 alone. Thus, TSA potentiates androgen-mediated PSA gene expression.

The same experiment was also performed in the presence of increasing amounts of Na-But or FR901288. As shown in Fig. 3B and C, both Na-But and FR901288 also increased R1881-induced PSA mRNA accumulation by about twofold. However, at higher concentrations, not only was this activation completely lost, but R1881- mediated mRNA accumulation was also effectively inhibited. Altogether, these data suggest that, as in the transient transfection experiments described above, HDACIs increase AR-dependent gene activation at its cellular target genes.

\section{E1A inhibits HDACI-mediated potentiation of AR activity}

It was previously shown that CBP, which has intrinsic HAT activity, can serve as a cofactor for AR (Aarnisalo et al. 1998, Frønsdal et al. 1998). To assess whether CBP is involved in the effect of HDACIs on AR activity, we 
A

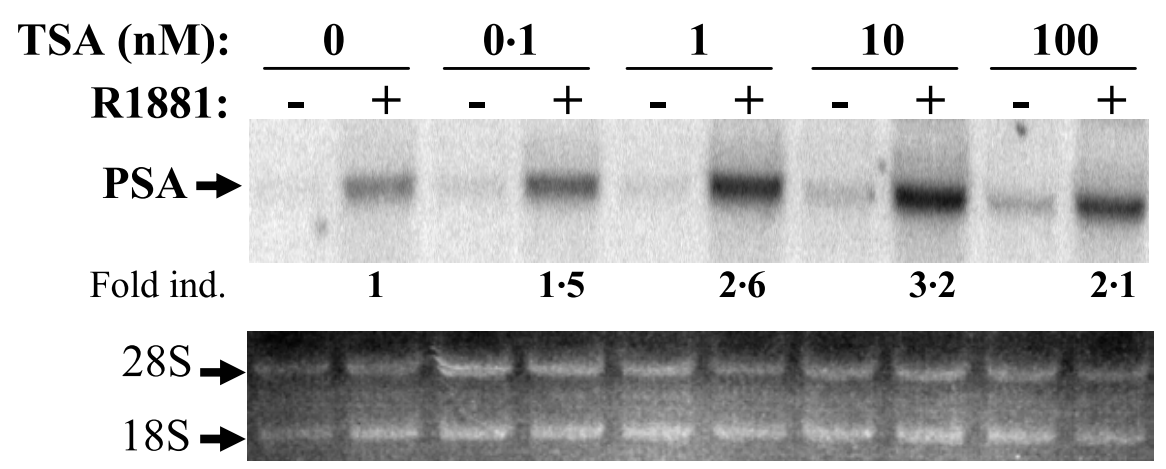

B
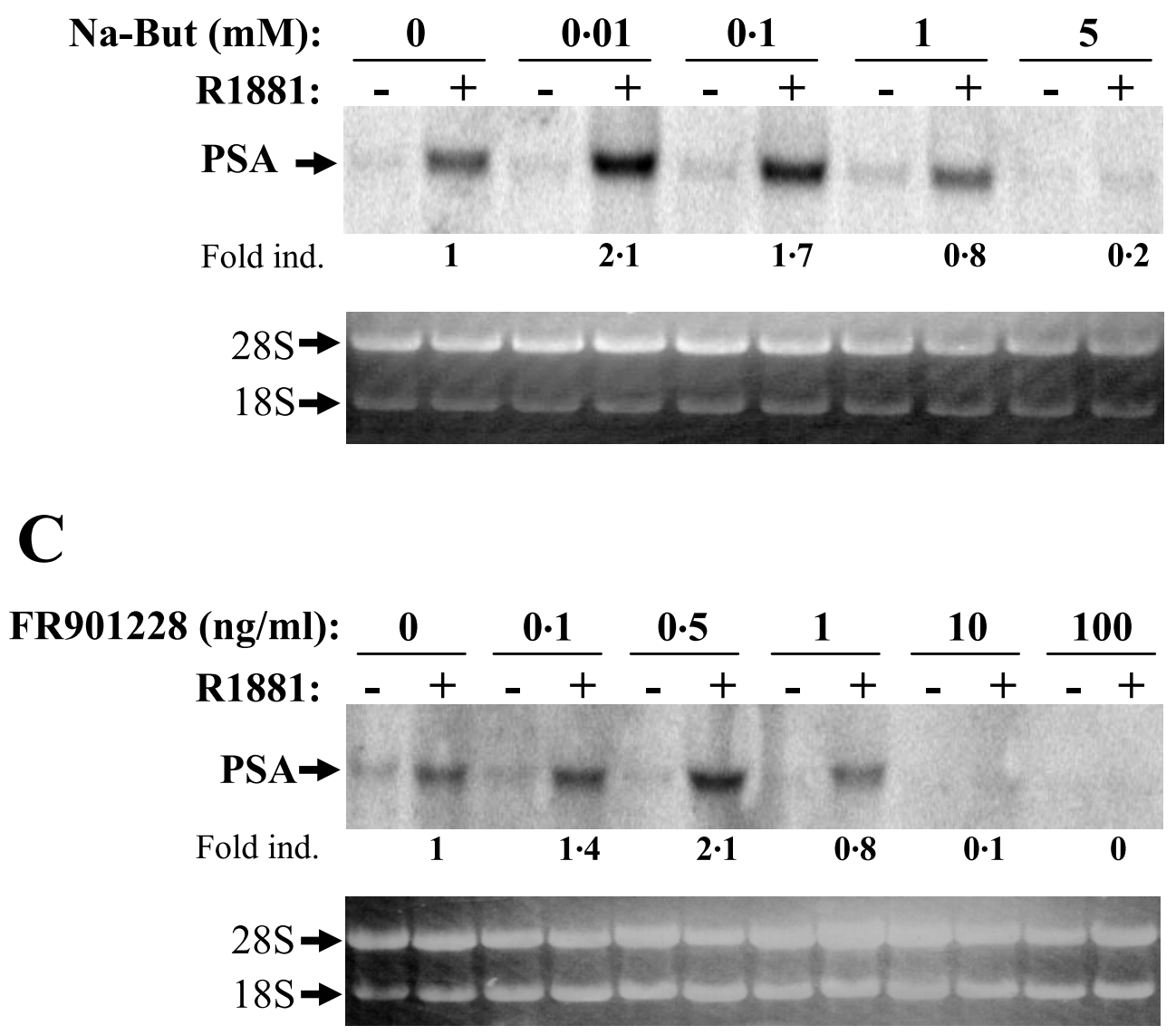

Figure 3 Histone deacetylase inhibitors increase PSA mRNA accumulation in LNCaP cells. (A) LNCaP cells were either left untreated or treated with R1881 $\left(10^{-7} \mathrm{M}\right)$ and/or with the indicated concentrations of TSA for $24 \mathrm{~h}$. Total RNA was isolated and was then used in Northern analysis with PSA as probe. EtBr-stained $28 \mathrm{~S}$ and $18 \mathrm{~S}$ tRNA bands are shown as controls. (B) Cells were treated similarly to panel A, but Na-But was used instead of TSA. Northern analysis was performed similarly to panel A. EtBr-stained 28S and 18S rRNA bands are shown. (C) Cells were treated with FR901228 instead of TSA as in panel A, and Northern analysis was performed. $28 \mathrm{~S}$ and $18 \mathrm{~S}$ tRNA bands are shown as control. 

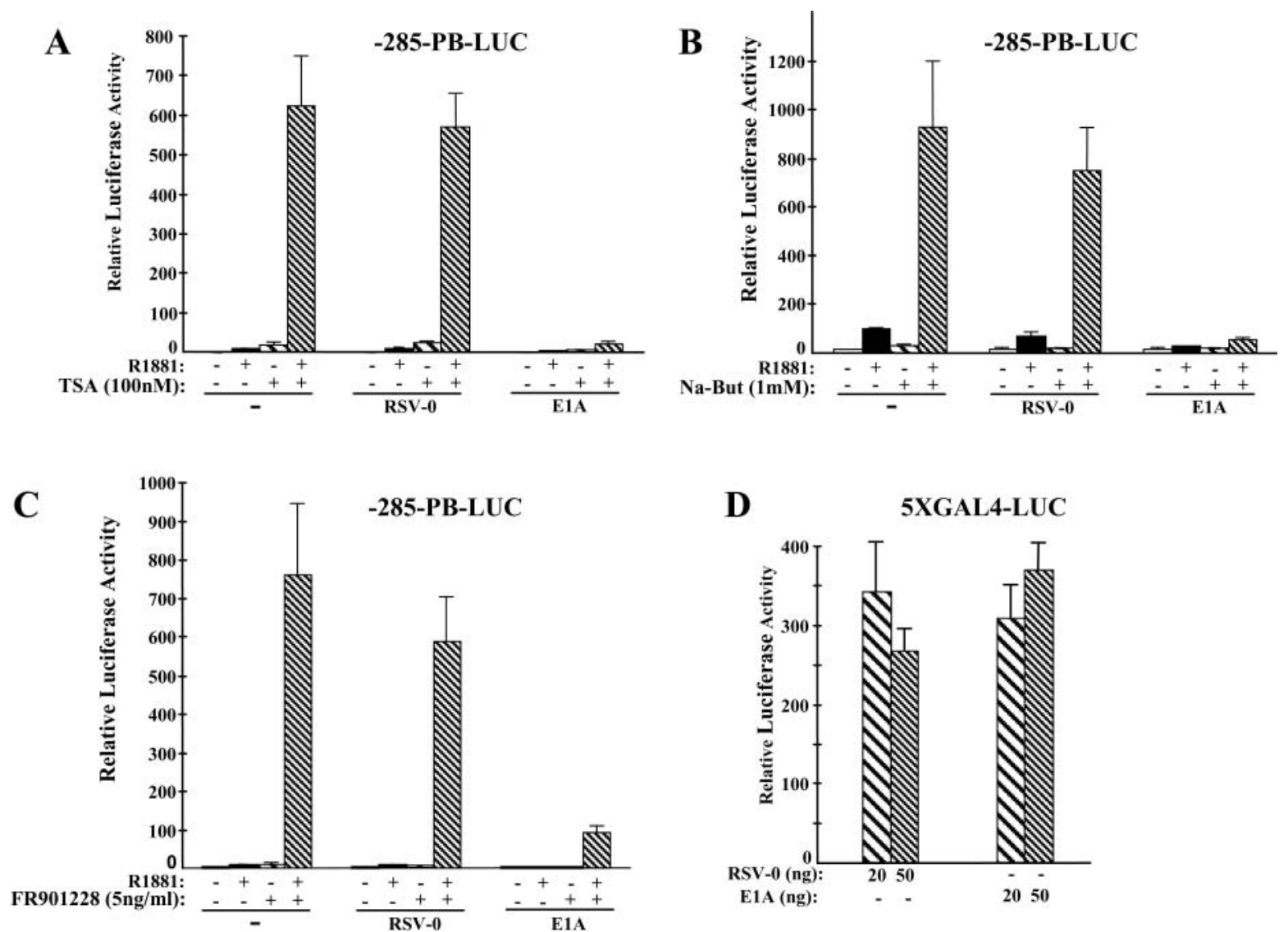

Figure 4 Inhibition of HDACl-potentiated AR-activity by E1A. (A) HeLa cells were transfected with the -285-PB-LUC reporter and pSG5-AR alone, or with the empty expression vector RSV-0 or the expression vector pBL9-E1A. Cells were either left untreated or treated with R1881 $\left(10^{-7} \mathrm{M}\right)$ and/or with $100 \mathrm{nM}$ of TSA and harvested after $18 \mathrm{~h}$, and LUC activities were determined. Activation of reporter gene expression in the presence of R1881, but in the absence of TSA, was arbitrarily set at 10 . Results represent the means of at least three independent experiments with S.E. indicated as error bars. (B) HeLa cells were similarly transfected as in panel A. Cells were either left untreated or treated with R1881 $\left(10^{-7} \mathrm{M}\right)$ and/or with $1 \mathrm{mM}$ of Na-But and harvested after $18 \mathrm{~h}$, and LUC activities were determined. Activation of reporter gene expression in the presence of R1881, but in the absence of TSA, was arbitrarily set at 100 . Results represent the means of at least three independent experiments with S.E. indicated as error bars. (C) HeLa cells were transfected as in panel A. Cells were either left untreated or treated with R1881 $\left(10^{-7} \mathrm{M}\right)$ and/or with $5 \mathrm{ng} / \mathrm{ml}$ of FR901228 and then harvested after $18 \mathrm{~h}$, and LUC activities were determined. Activation of reporter gene expression in the presence of R1881, but in the absence of FR901228, was arbitrarily set at 10 . Results represent the means of at least three independent experiments with S.E. indicated as error bars. (D) HeLa cells were transfected with the 5XGAL4-LUC reporter and $10 \mathrm{ng}$ of pSG5-GAL4 expression plasmid. The effect of cotransfection of an empty expression plasmid, RSV-0, or the expression vector for E1A, as indicated at the bottom of the Figure, is shown. Results shown are from an experiment done in triplicate which was repeated two times with comparable results. S.E. is indicated as error bars.

used the viral oncoprotein E1A, which is known to inhibit CBP activity (Chakravarti et al. 1999, Li et al. 1999, Perissi et al. 1999). We performed a series of transient transfection assays in HeLa cells. -285-PB-LUC was cotransfected with $A R$ and either an empty expression vector or a vector encoding E1A, followed by hormone and HDACI treatments at optimal concentrations for the potentiation of AR-mediated transcription. As in the experiments described in Fig. 1 and 2, TSA increased the R1881-induced levels of reporter expression by 60-fold. Whereas in the presence of an empty expression vector, similar results were obtained, when E1A was ectopically expressed, nearly all of the TSA effect was lost (Fig. 4A). As expected, E1A expression also abrogated the effect of R1881 on the reporter activity, suggesting that CBP directly contributes to AR activity in HeLa cells. Essentially identical results were obtained when Na-But or FR901228 was used (Fig. 4B and C); in contrast, E1A had no effect on 5XGAL4-LUC (Sadowski \& Ptashne 1989), a reporter construct known not to be affected by AR or CBP (Fig. 4D). These data suggest that E1A blocks HDACI-mediated increase in AR activity. 


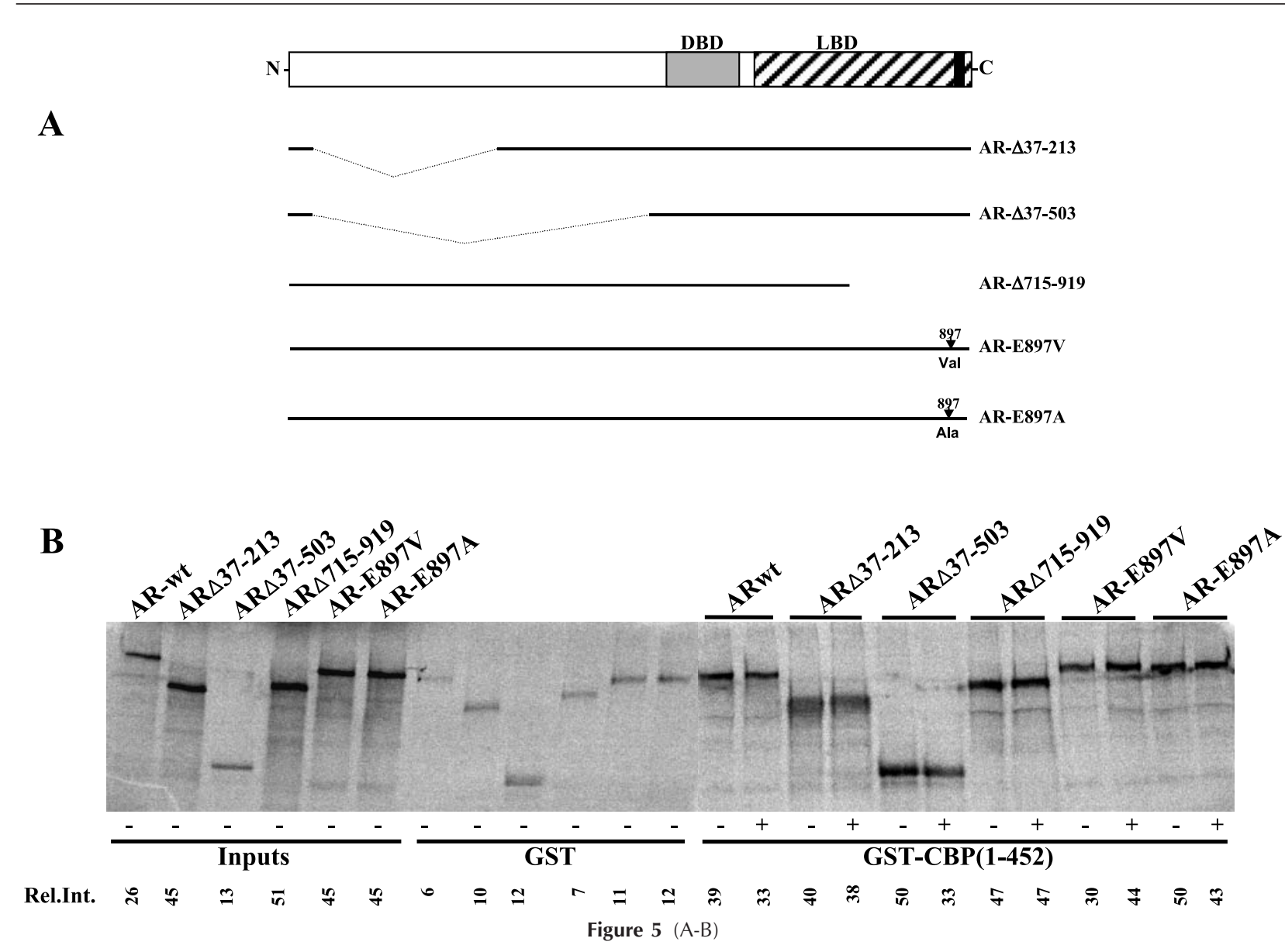

Figure 5 Mapping the domains of AR involved in the HDACI responses. (A) Schematic depiction of the different mutants of AR that were used in the studies described below. (B) GST pull-down assay, where in vitro translated wild-type AR and the AR mutants were tested for their ability to interact with GST-CBP(1-452) or GST as control. Note that there was underloading in the gel for the ARA37-503 mutant for the input. (C) HeLa cells were transfected with the -285-PB-LUC reporter, wild-type AR or the different AR mutants. Cells were either left untreated or treated with R1881 $\left(10^{-9} \mathrm{M}\right)$ and/or with TSA $(100 \mathrm{nM})$ and harvested after $18 \mathrm{~h}$, and LUC activities were determined. Activation of reporter gene expression in the presence of wild-type AR and R1881, but in the absence of TSA, was arbitrarily set at $10 \%$. Results represent the means of at least three independent experiments with S.E. indicated as error bars. (D) HeLa cells were transfected as in panel C. Cells were either left untreated or treated with R1881 (10 $\left.{ }^{-7} \mathrm{M}\right)$ and/or with Na-But $(1 \mathrm{mM})$ and harvested after $18 \mathrm{~h}$, and LUC activities were determined. Activation of reporter gene expression in the presence of wild-type AR and R1881, but in the absence of Na-But, was arbitrarily set at $10 \%$. Results represent the means of at least three independent experiments with S.E. indicated as error bars. (E) HeLa cells were transfected with the -285-PB-LUC reporter, wild-type AR or the different AR mutants, in the presence or absence of CMV-CBP $(100 \mathrm{ng})$. Cells were left untreated or treated with R1881 (10 $\left.{ }^{-7} \mathrm{M}\right)$ and harvested after $18 \mathrm{~h}$, and LUC activities were determined. Activation of reporter gene expression in the presence of wild-type AR and R1881, but in the absence of CBP, was arbitrarily set at 100. Results represent the means of at least three independent experiments with S.E. indicated as error bars.

Domains of $A R$ that are involved in activation by HDACIs coincide with those that respond to CBP

We next investigated the regions of AR that contribute to its activation by TSA and $\mathrm{Na}$-But and whether there was a correlation between the domains required for HDACI response and activation by CBP. To that end, we used two $\mathrm{N}$-terminal internal deletion mutants (Slagsvold et al. 2000) and a C-terminal deletion mutant (Frønsdal et al. 1998), as well as two point mutants in the AF-2 core that have significantly impaired AR function (Slagsvold et al. 2000) (Fig. 5A). We first tested the ability of these AR mutants to interact with CBP in the GST pull-down assay, as described previously (Frønsdal et al. 1998). As shown in Fig. 5B, all AR mutants bound CBP similarly to wild-type $A R$, suggesting that any difference in activity is not caused by changes due to lack of CBP interaction.

These mutants were then tested in transient transfection assays in HeLa cells to assess their transactivation properties in response to TSA or Na-But. -285-PB-LUC was 
C
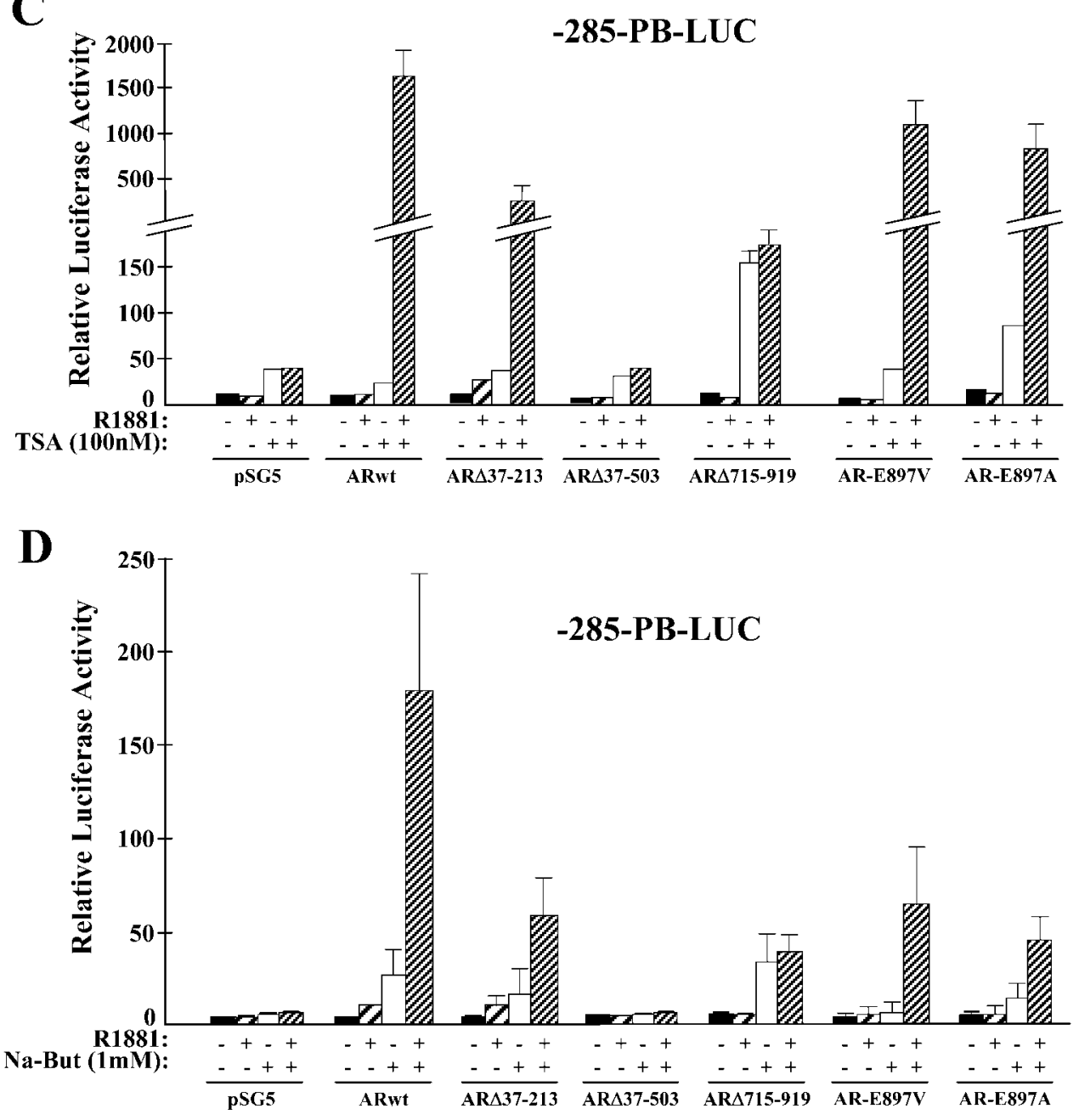

$\mathbf{E}$

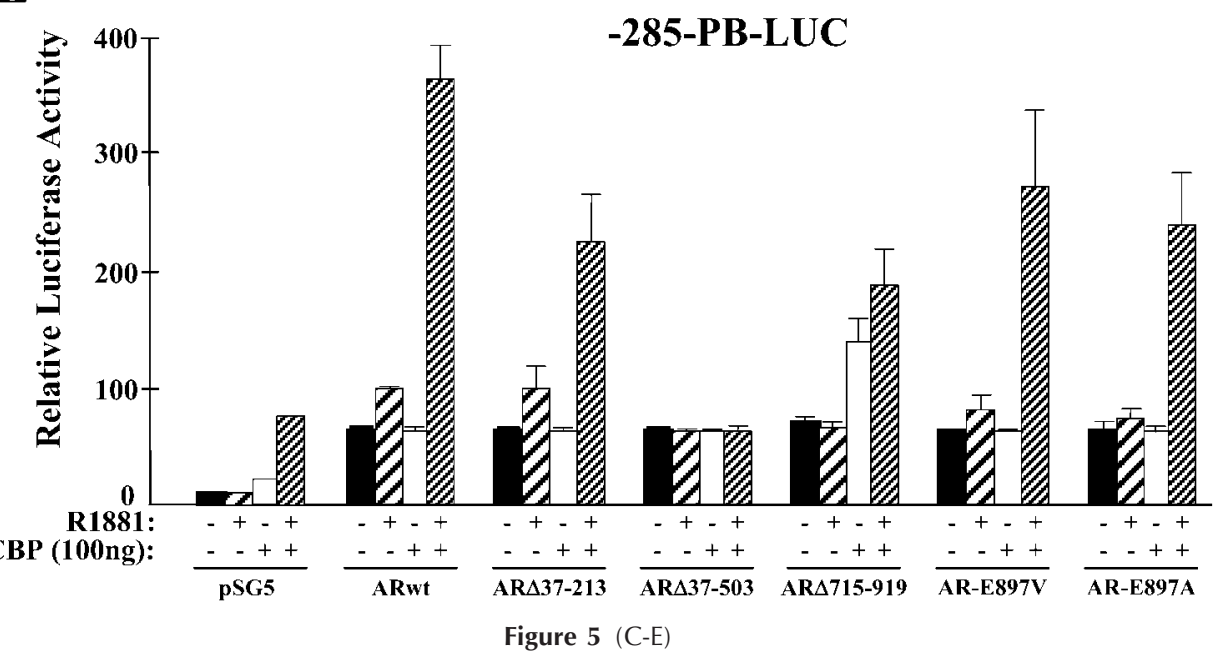


cotransfected into HeLa cells with either the empty expression vector pSG5 or expression vectors specifying wild-type AR or its mutants. After transfection, cells were either left untreated or treated with the synthetic androgen R1881 and/or with TSA or Na-But. After 18 h, cells were harvested and LUC activities were determined. As shown in Fig. 5C, an approximately 150-fold increase from basal level was observed when cells were transfected with wild-type AR and treated with both R1881 and TSA. Significant but lower activation was also observed for all the other mutants, but not for $\mathrm{AR} \Delta 37-503$ in which most of the N-terminus of AR has been removed (Fig. 5A). As expected, the AR $\Delta 715-919$ mutant did not respond to R1881, since a big portion of the LBD is missing, but its activity was significantly increased by TSA. The differences in activity were not due to differential expression of the proteins determined by Western analysis (data not shown). These results suggest that an intact $\mathrm{N}$-terminal domain is required for AR to respond to HDACIs, and that the AF-2 domain does not play a significant role in this process.

In a similar experiment using $\mathrm{Na}$-But, qualitatively comparable results were obtained (Fig. 5D). AR $\Delta 37-503$ completely lost the ability to respond to $\mathrm{Na}-\mathrm{But}$, whereas the other mutants had approximately $20-30 \%$ of wild-type activity. These data corroborate the findings with TSA and point to the $\mathrm{N}$-terminus of $\mathrm{AR}$ as the most important region involved in mediating the effects of $\mathrm{Na}-\mathrm{But}$ on $\mathrm{AR}$ activity.

We then tested the ability of these mutants to respond to CBP in the transient transfection assay. $-285-\mathrm{PB}-\mathrm{LUC}$ was cotransfected into HeLa cells as above, with either an empty expression vector or expression vectors specifying wild-type AR or its mutants in the presence or absence of an expression vector for CBP. After transfection, cells were either left untreated or treated with R1881; after $18 \mathrm{~h}$, cells were harvested and LUC activities were determined. As shown in Fig. 5E, the response of the various mutants to $\mathrm{CBP}$ was qualitatively similar to their response to HDACIs. Whereas CBP increased wild-type AR activity by nearly fourfold, no activation could be detected with AR $\Delta 37-503$. For the other mutants, the CBP response ranged between two- and threefold. Altogether, the strong correlation between the response of $\mathrm{AR}$ mutants to HDACIs and CBP suggests that the HAT activity of CBP is involved in regulating AR transactivation potential.

\section{Discussion}

Recent studies have documented the involvement of histone acetylation of nuclear receptor target promoters in ligand-regulated modulation of transcription. This was first suggested by the identification of nuclear receptor coactivators with intrinsic HAT activity or recruitment by coactivators of proteins with HAT activity, as well as nuclear receptor corepressors that associate with HDACs (for reviews, see Glass \& Rosenfeld 2000, McKenna \& O'Malley 2002). Subsequent studies suggested that changes in the histone acetylation status of cells may have an important role in nuclear receptor activity. Consistent with this notion, a recent study analyzing the factors that bind to the PSA gene promoter and enhancer regions in vivo identified recruitment of coactivators with HAT activity in the presence of agonists and recruitment of corepressors and HDACs in the presence of antagonists (Shang et al. 2002).

We have systematically studied the role of the histone acetylation status of the cell in AR function. All three independent HDACIs used in this study increased endogenous AR target gene expression and the activity of AR-dependent reporter constructs. Mapping of the region in AR that responds to the HDACIs suggests that it is the $\mathrm{N}$-terminal domain that is most important for mediating this response.

The possible role of histone acetylation in AR function was first suggested several years ago when CBP, which has intrinsic HAT activity and can recruit HAT-containing proteins to target promoters (for a review, see Chan \& La Thangue 2001), was found to be a coactivator for AR (Aarnisalo et al. 1998, Frønsdal et al. 1998). In the current study, we found very good correlation between the ability of various mutants of AR to respond to HDACI treatment and $\mathrm{CBP}$ coexpression. In addition, ectopic E1A expression, which is known to inhibit CBP activity (Chakravarti et al. 1999, Li et al. 1999, Perissi et al. 1999), essentially annihilated the stimulatory effects on AR transactivation potential of all three HDACIs tested. These results suggest that CBP, or an associated factor, may be involved in providing the HAT activity that is necessary to activate AR target genes. The interactions of AR with CBP may be direct or mediated by an intermediary cofactor bridging the two factors. Further work using HAT mutants of CBP would shed more light on this issue.

Although all three HDACIs used in this study had similar qualitative effects on the endogenous PSA promoter and the AR-dependent reporter constructs, there were quantitative differences. First, TSA was the strongest of the HDACIs in increasing AR activity both for the PSA transcription and at the AR-dependent reporter construct -285-PB-LUC, requiring only $\mathrm{nM}$ amounts to give rise to the highest fold activation. Second, whereas at high concentrations of TSA there was only a small decrease in their efficacy, Na-But and FR901228 strongly inhibited endogenous PSA expression when used at concentrations that are optimal for the activation of the -285-PB-LUC. There may be several reasons to explain these observations. First, TSA is known to be significantly more potent than other HDACIs (de Ruijter et al. 2003), as consistent with our data. Second, there appear to be differences in the efficiency with which the different HDACIs interact with and inhibit the activity of different HDACs. For example, 
whereas TSA effectively blocks HDAC4, Na-But is significantly less efficient in this regard (de Ruijter et al. 2003). Thus, the reason for the observed differences not only might lie in the variation in chromatin structure between the PSA promoter in LNCaP cells and the probasin promoter in $\mathrm{LNCaP}$ or $\mathrm{HeLa}$ cells (giving rise to differences in sensitivity to HDACIs), but could also be related to the HDAC(s) these two promoters are associated with. This is also supported by our finding that whereas FR901228 and, to a lesser extent, Na-But increase the activation of the AR-expression plasmid in transfected cells, especially in the absence of hormone, TSA did not have such an effect. However, this increased AR expression could also be due to the nonspecific effects of $\mathrm{Na}$-But previously reported (Thiagalingam et al. 2003). FR 901228 could potentially have similar nonspecific effects due to the $\mu \mathrm{M}$ amounts required for this compound in the experiments. Further work is needed to assess these possibilities.

Mutational analysis of AR indicated that the effects of HDACIs were directed primarily at the $\mathrm{N}$-terminus of AR. However, since the HDACI effects are hormone dependent, an intact LBD is also required for maximal activity. Interestingly, hormone-binding-capable, but transcriptionally compromised AF-2 mutants of AR (Slagsvold et al. 2000) were rescued almost to wild-type levels by TSA, and up to $30 \%$ of wild-type levels by Na-But. This rescue phenomenon coincided with the ability of ectopically expressed CBP to rescue the AR mutants. It has been suggested that the AF- 2 core domain, where the mutants that we tested reside, has an important role in the intramolecular interaction between the $\mathrm{N}$-terminal domain and the LBD of AR that is necessary for transcriptional activation by AR (Slagsvold et al. 2000, $\mathrm{He} \&$ Wilson 2002). It has also been suggested that coactivators, such as CBP, could be involved in mediating this intramolecular interaction. It is therefore tempting to speculate that HDACIs may have a role in facilitating this interaction, in addition to their roles in chromatin packing through changes in histone acetylation. Further work would be required to test this possibility.

Previous studies used TSA or $\mathrm{Na}-\mathrm{But}$ to examine the effect of histone acetylation on AR activity (List et al. 1999, Sadar \& Gleave 2000). TSA was found to increase activation of the mouse mammary tumor virus (MMTV) promoter in chromatin by $\mathrm{AR}$, at least in part by an increase in AR levels in 29+ L-cell fibroblasts (List et al. 1999). In another study, Na-But did not affect the total levels of AR in LNCaP cells, but increased intranuclear localization of AR (Sadar \& Gleave 2000). Under the same conditions of the transient transfection experiments that tested the effects of HDACIs on AR activity, we did not find any significant change in AR protein levels, either total, nuclear or cytoplasmic, in the presence or absence of TSA, Na-But or FR901228, independently of the presence or absence of androgens (Fig. 1C and data not shown). Our findings, therefore, do not support the hypothesis that the effect of HDACIs on AR activity in LNCaP cells is due to an increase in AR protein levels. In the case of the ectopically expressed AR in HeLa cells, the effect of Na-But and FR901228, but not TSA, may partly be mediated by changes in AR levels (Fig. 2C). To determine whether this is due to changes in the nature of proteins associated with the expression plasmid promoter, or is simply due to nonspecific effects of these compounds, will require further analysis.

Our data are most consistent with an alternative hypothesis. The robust increase in AR-transactivation potential in the presence of HDACIs suggests that an increase in histone acetylation may have a direct role in modulating AR activity. It is possible that HDACIs prepare the target promoter to assume a more accessible conformation to which hormone-bound AR and associated proteins can bind and activate transcription to higher levels than when the HDACIs are not present. However, in future studies, it is important to assess any other modifications in the histones, as well as the acetylation state of the AR itself, which has recently been suggested to contribute to its transactivation potential (Fu et al. 2000, 2003, Gaughan et al. 2002).

HDACIs are being evaluated for use in differentiation therapy of cancer, including prostate cancer (Samid et al. 1997, Conley et al. 1998, Huang et al. 1999). Given the important role of androgens in the initial stages of prostate cancer, as well as the deregulated expression of AR target genes in the advanced, androgen-independent phase of prostate cancer (Gregory et al. 1998, Korkmaz et al. 2000), the future elucidation of the molecular mechanism of HDACI effects on androgen-regulated genes may provide critical new information on both normal AR function and the progression of prostate cancer.

\section{Acknowledgements}

We thank Dr Jorma Palvimo for providing the -285-PBLUC reporter plasmid and Dr Robert Schultz for the gift of FR901228. This work was supported by grants from the Norwegian Research Council and Norwegian Cancer Society to FS.

\section{References}

Aarnisalo P, Palvimo JJ \& Janne OA 1998 CREB-binding protein in androgen receptor-mediated signaling. PNAS 95 2122-2127.

Bannister AJ \& Kouzarides T 1996 The CBP co-activator is a histone acetyltransferase. Nature 384 641-643.

Chakravarti D, Ogryzko V, Kao HY, Nash A, Chen H, Nakatani Y \& Evans RM 1999 A viral mechanism for inhibition of p300 and PCAF acetyltransferase activity. Cell 96 393-403.

Chan HM \& La Thangue NB 2001 p300/CBP proteins: HATs for transcriptional bridges and scaffolds. Journal of Cell Science 114 2363-2373. 
Conley BA, Egorin MJ, Tait N, Rosen DM, Sausville EA, Dover G, Fram RJ \& Van Echo DA 1998 Phase I study of the orally administered butyrate prodrug, tributyrin, in patients with solid tumors. Clinical Cancer Research 4 629-634.

Cress WD \& Seto E 2000 Histone deacetylases, transcriptional control, and cancer. Journal of Cellular Physiology 184 1-16.

Deleu L, Shellard S, Alevizopoulos K, Amati B \& Land H 2001 Recruitment of TRRAP required for oncogenic transformation by E1A. Oncogene 20 8270-8275.

de Ruijter AJ, van Gennip AH, Caron HN, Kemp S \& van Kuilenburg AB 2003 Histone deacetylases (HDACs): characterization of the classical HDAC family. Biochemical Journal $370737-749$.

Doesburg P, Kuil CW, Berrevoets CA, Steketee K, Faber PW, Mulder E, Brinkmann AO \& Trapman J 1997 Functional in vivo interaction between the amino-terminal, transactivation domain and the ligand binding domain of the androgen receptor. Biochemistry 36 1052-1064.

Frønsdal K, Engedal N, Slagsvold T \& Saatcioglu F 1998 CREB binding protein is a coactivator for the androgen receptor and mediates cross-talk with AP-1 Journal of Biological Chemistry 273 31853-31859 [erratum 1999274 25188].

Frønsdal K, Engedal N \& Saatcioglu F 2000 Efficient DNA-mediated gene transfer into prostate cancer cell line LNCaP. Prostate $\mathbf{4 3}$ 111-117.

Fu M, Wang C, Reutens AT, Wang J, Angeletti RH, Siconolfi-Baez L, Ogryzko V, Avantaggiati ML \& Pestell RG 2000 p300 and p300/cAMP-response element-binding protein-associated factor acetylate the androgen receptor at sites governing hormone-dependent transactivation. Journal of Biological Chemistry 275 20853-20860.

Fu M, Rao M, Wang C, Sakamaki T, Wang J, Di Vizio D, Zhang X, Albanese C, Balk S, Chang C et al. 2003 Acetylation of androgen receptor enhances coactivator binding and promotes prostate cancer cell growth. Molecular and Cellular Biology 23 8563-8575.

Gaughan L, Logan IR, Cook S, Neal DE \& Robson CN 2002 Tip60 and histone deacetylase 1 regulate androgen receptor activity through changes to the acetylation status of the receptor. Journal of Biological Chemistry 277 25904-25913.

Glass CK \& Rosenfeld MG 2000 The coregulator exchange in transcriptional functions of nuclear receptors. Genes and Development 14 121-141.

Goodman RH \& Smolik S 2000 CBP/p300 in cell growth, transformation, and development. Genes and Development 14 1553-1577.

Gregory CW, Hamil KG, Kim D, Hall SH, Pretlow TG, Mohler JL \& French FS 1998 Androgen receptor expression in androgen-independent prostate cancer is associated with increased expression of androgen-regulated genes. Cancer Research $\mathbf{5 8}$ $5718-5724$.

Gu W \& Roeder RG 1997 Activation of p53 sequence-specific DNA binding by acetylation of the p53 C-terminal domain. Cell $\mathbf{9 0}$ 595-606.

He B \& Wilson EM 2002 The NH(2)-terminal and carboxyl-terminal interaction in the human androgen receptor. Molecular Genetics and Metabolism 75 293-298.

Horoszewicz JS, Leong SS, Kawinski E, Karr JP, Rosenthal H, Chu TM, Mirand EA \& Murphy GP 1983 LNCaP model of human prostatic carcinoma. Cancer Research 43 1809-1818.

Huang H, Reed CP, Zhang JS, Shridhar V, Wang L \& Smith DI 1999 Carboxypeptidase A3 (CPA3): a novel gene highly induced by histone deacetylase inhibitors during differentiation of prostate epithelial cancer cells. Cancer Research 59 2981-2988.

Hung HL, Lau J, Kim AY, Weiss MJ \& Blobel GA 1999 CREB-binding protein acetylates hematopoietic transcription factor GATA-1 at functionally important sites. Molecular and Cellular Biology 19 3496-3505.
Ikonen T, Palvimo JJ \& Janne OA 1997 Interaction between the amino- and carboxyl-terminal regions of the rat androgen receptor modulates transcriptional activity and is influenced by nuclear receptor coactivators. Journal of Biological Chemistry 272 29821-29828.

Jenster G, Spencer TE, Burcin MM, Tsai SY, Tsai MJ \& O’Malley BW 1997 Steroid receptor induction of gene transcription: a two-step model. PNAS 94 7879-7884.

Korkmaz KS, Korkmaz CG, Ragnhildstveit E, Pretlow TG \& Saatcioglu F 2000 An efficient procedure for cloning hormone-responsive genes from a specific tissue. DNA and Cell Biology 19 499-506.

Kramer OH, Gottlicher M \& Heinzel T 2001 Histone deacetylase as a therapeutic target. Trends in Endocrinology and Metabolism 12 294-300.

Kwok RP, Lundblad JR, Chrivia JC, Richards JP, Bachinger HP, Brennan RG, Roberts SG, Green MR \& Goodman RH 1994 Nuclear protein CBP is a coactivator for the transcription factor CREB. Nature 370 223-226.

Li Q, Imhof A, Collingwood TN, Urnov FD \& Wolffe AP 1999 p300 stimulates transcription instigated by ligand-bound thyroid hormone receptor at a step subsequent to chromatin disruption. EMBO Journal 18 5634-5652.

Lindzey J, Kumar MV, Grossman M, Young C \& Tindall DJ 1994 Molecular mechanisms of androgen action. Vitamins and Hormones $49383-432$.

List HJ, Smith CL, Rodriguez O, Danielsen M \& Riegel AT 1999 Inhibition of histone deacetylation augments dihydrotestosterone induction of androgen receptor levels: an explanation for trichostatin A effects on androgen-induced chromatin remodeling and transcription of the mouse mammary tumor virus promoter. Experimental Cell Research 252 471-478.

Lundwall A \& Lilja H 1987 Molecular cloning of human prostate specific antigen cDNA. FEBS Letters 214 317-322.

Mangelsdorf DJ, Thummel C, Beato M, Herrlich P, Schutz G, Umesono K, Blumberg B, Kastner P, Mark M, Chambon P et al. 1995 The nuclear receptor superfamily: the second decade. Cell $\mathbf{8 3}$ 835-839.

Marks PA, Richon VM \& Rifkind RA 2000 Histone deacetylase inhibitors: inducers of differentiation or apoptosis of transformed cells. Journal of the National Cancer Institute 92 1210-1216.

McInerney EM, Tsai MJ, O’Malley BW \& Katzenellenbogen BS 1996 Analysis of estrogen receptor transcriptional enhancement by a nuclear hormone receptor coactivator. PNAS 93 10069-10073.

McKenna NJ \& O'Malley BW 2002 Minireview: nuclear receptor coactivators - an update. Endocrinology 143 2461-2465.

Newmark HL, Lupton JR \& Young CW 1994 Butyrate as a differentiating agent: pharmacokinetics, analogues and current status. Cancer Letters 78 1-5.

Ogryzko VV, Schiltz RL, Russanova V, Howard BH \& Nakatani Y 1996 The transcriptional coactivators p300 and CBP are histone acetyltransferases. Cell 87 953-959.

Perissi V, Dasen JS, Kurokawa R, Wang Z, Korzus E, Rose DW, Glass CK \& Rosenfeld MG 1999 Factor-specific modulation of CREB-binding protein acetyltransferase activity. PNAS 96 3652-3657.

Quigley CA, De Bellis A, Marschke KB, el-Awady MK, Wilson EM \& French FS 1995 Androgen receptor defects: historical, clinical, and molecular perspectives. Endocrine Reviews 16 271-321 [erratum 16 546].

Sadar MD \& Gleave ME 2000 Ligand-independent activation of the androgen receptor by the differentiation agent butyrate in human prostate cancer cells. Cancer Research 60 5825-5831.

Sadowski I \& Ptashne M 1989 A vector for expressing GAL4(1-147) fusions in mammalian cells. Nucleic Acids Research 177539.

Sakaguchi K, Herrera JE, Saito S, Miki T, Bustin M, Vassilev A, Anderson CW \& Appella E 1998 DNA damage activates p53 through a phosphorylation-acetylation cascade. Genes and Development 12 2831-2841. 
Samid D, Hudgins WR, Shack S, Liu L, Prasanna P \& Myers CE 1997 Phenylacetate and phenylbutyrate as novel, nontoxic differentiation inducers. Advances in Experimental Medicine and Biology 400 501-505.

Shang Y, Myers M \& Brown M 2002 Formation of the androgen receptor transcription complex. Molecular Cell 9 601-610.

Slagsvold T, Kraus I, Bentzen T, Palvimo J \& Saatcioglu F 2000 Mutational analysis of the androgen receptor AF-2 (activation function 2) core domain reveals functional and mechanistic differences of conserved residues compared with other nuclear receptors. Molecular Endocrinology 14 1603-1617.

Thiagalingam S, Cheng KH, Lee HJ, Mineva N, Thiagalingam A \& Ponte JF 2003 Histone deacetylases: unique players in shaping the epigenetic histone code. Annals of the New York Academy of Sciences 983 84-100.

Tsai MJ \& O’Malley BW 1994 Molecular mechanisms of action of steroid/thyroid receptor superfamily members. Annual Review of Biochemistry 63 451-486.
Tsuji N, Kobayashi M, Nagashima K, Wakisaka Y \& Koizumi K 1976 A new antifungal antibiotic, trichostatin. Journal of Antibiotics (Tokyo) 29 1-6.

Xi Z, Klokk TI, Korkmaz K, Kurys P, Elbi C, Risberg B, Danielsen H, Loda M \& Saatcioglu F 2004 Kallikrein 4 is a predominantly nuclear protein and is overexpressed in prostate cancer. Cancer Research 64 2365-2370.

Yoshida M, Kijima M, Akita M \& Beppu T 1990 Potent and specific inhibition of mammalian histone deacetylase both in vivo and in vitro by trichostatin A. Journal of Biological Chemistry 265 17174-17179.

Young CY, Andrews PE \& Tindall DJ 1995 Expression and androgenic regulation of human prostate-specific kallikreins. Journal of Andrology 16 97-99.

Received 11 May 2004

Accepted 8 June 2004 